\title{
Cortical Atrophy, White Matter Lesions, and Bulb Configuration in Patients with Idiopathic Olfactory Loss and Other Causes of Olfactory Loss
}

\author{
Alexander Wieck Fjaeldstad ${ }^{a, b}$ Therese Ovesen ${ }^{a, b} \quad$ Rikke Beese Dalby ${ }^{c}$ \\ aDepartment of Otorhinolaryngology, Flavour Clinic, Regional Hospital West Jutland, Holstebro, Denmark; ${ }^{b}$ Flavour \\ Institute, Aarhus University, Aarhus, Denmark; 'Section of Neuroradiology, Department of Radiology, Aarhus \\ University Hospital, Aarhus, Denmark
}

\section{Keywords}

Magnetic resonance imaging $\cdot$ Rhinology $\cdot$ Smell

\begin{abstract}
Introduction: While magnetic resonance imaging (MRI) is not included in the current guidelines for diagnosing olfactory disorders in the most recent position paper on olfactory dysfunction, both 1.5T and 3T MRI are commonly used in the diagnostic workup of many patients with olfactory loss. Often, MRI is used to rule out intracranial tumours, but other useful information may be obtained from MRI scans in these patients. The potential of MRI in olfactory loss depends on sufficient knowledge of structural changes in different aetiologies of olfactory loss. We present common clinical MRI findings in olfactory loss and evaluate the usefulness of structural integrity scores in differentiating between aetiologies. Methods: In this study, we investigated if white matter hyperintensities (WMHs, measured by Fazekas score), global cortical atrophy (GCA), and medial temporal lobe atrophy (MTA) are more common in patients with idiopathic olfactory loss than in patients with acquired olfactory loss due to other aetiologies. Furthermore, we compared olfactory bulb (OB) configurations in different olfactory loss aetiologies. Results: In 88 patients with olfactory loss, WMHs, GCA, and MTA were not more significant findings on MRI in idiopathic olfac-
\end{abstract}

karger@karger.com

(c) 2021 S. Karger AG, Basel

www.karger.com/orl

Karger" tory loss $(n=51)$ compared with other causes of acquired olfactory loss (Fazekas score $p=0.2977$; GCA score $p=0.6748$; MTA score $p=0.7851$ ). Bulb configurations differed in patients suffering from post-traumatic olfactory loss and may aid in identifying the underlying aetiology in patients where trauma is among the suspected causes of olfactory loss. Conclusion: We recommend that structural MRI with an OB sequence is included in the diagnostic evaluation of olfactory loss with suspected congenital and post-traumatic aetiology and should be considered in idiopathic olfactory loss with suspected central aetiology (e.g., tumour).

(c) 2021 S. Karger AG, Basel

\section{Background}

While the quantification of olfactory loss is assessed by application of validated psychophysical olfactory tests, determining the underlying cause can prove more difficult. Conclusions on the underlying cause is established by combining information on the patient's medical history, the nature and chronology of the olfactory impairment, accompanying symptoms, clinical examination, and paraclinical testing. In spite of a full clinical workup, a fourth of patients referred with olfactory impairment have negative findings for the most common causes of 
olfactory disorders (sinonasal disease, infections of the upper respiratory tract, head trauma, and toxic exposure) and are diagnosed with idiopathic olfactory loss as no clear underlying aetiology can be found $[1,2]$.

The current guidelines for the diagnosis of olfactory disorders do not include magnetic resonance imaging (MRI) in the standard clinical algorithm [3]. The current level of evidence for choosing to perform MRI in patients with olfactory dysfunction is based on case series and expert opinion [4]. A main focus of diagnostic MRI scans in patients with olfactory loss is to rule out intracranial tumours, a rare potential underlying cause of olfactory loss [5]. Meanwhile, the clinical relevance of many other cerebral abnormalities in the diagnostic workup of patients with olfactory loss has previously been sparsely investigated.

An important differential diagnosis in patients with idiopathic olfactory loss is neurodegenerative disease. Olfactory dysfunction is a common prodromal manifestation in various neurodegenerative conditions, including Parkinson's disease, Alzheimer's disease, Lewy body disease, multiple sclerosis, posterior cortical atrophy, and multiple system atrophy $[6,7]$. In a large follow-up study, $9.8 \%$ of patients with idiopathic olfactory loss had been diagnosed with Parkinson's disease 10.9 years after olfactory loss onset [8]. In Parkinson's disease, cortical atrophy in olfactory-related brain regions is correlated with olfactory dysfunction $[9,10]$. Olfactory loss is associated with loss of both grey and white matter volume in several cortical areas [11-13]. In Alzheimer's disease and mild cognitive impairment, olfactory dysfunction is associated with the degree of medial temporal lobe atrophy (MTA) [14]. Hence, assessment of cortical atrophy may be relevant in patients with idiopathic olfactory loss to support at diagnosis of underlying neurodegenerative disease. Although the diagnosis of idiopathic smell loss entails a high risk of underlying neurodegenerative pathology, it is currently not known if idiopathic smell loss is associated with higher occurrence of cortical atrophy.

While the association between olfactory function and olfactory bulb (OB) volume has been well described in previous studies [15], information on different bulb configurations is sparse [16]. In a recent study, Lu et al. [17] report an association between olfactory function and volumes of central olfactory structures in older age groups, primarily mediated through $\mathrm{OB}$ volume. Even though these morphological differences have recently been proposed to provide diagnostic information on different aetiologies of olfactory dysfunction [18], no overview of bulb variations or classification system currently exists.
A common finding on MRI, with increasing incidence in older age, is cerebral white matter lesions, seen as white matter hyperintensities (WMHs) on T2-weighted MRI of the brain of presumed vascular origin [19]. In patients with mild cognitive impairment, olfactory test scores have been associated with cerebral white matter lesions, independently from cortical atrophy of olfactory regions [20]. The load of white matter abnormalities has also been found to correlate with olfactory loss in patients with multiple sclerosis [21].

The aim of this study is to investigate if common MRI findings such as WHMs, global cortical atrophy (GCA), and MTA is more frequent in patients with idiopathic olfactory loss than in patients with acquired olfactory loss due to other aetiologies. This may imply the inclusion of structural MRI to address these imaging features in future guidelines for diagnosing idiopathic olfactory loss. Furthermore, the aim is to describe different configurations of the OBs and to investigate if these differ between patients with different causes of olfactory loss.

\section{Methods}

\section{Patients}

Patients with olfactory loss were referred to the Flavour Clinic, Holstebro, Central Denmark Region, Denmark; the clinic receive patients from private otolaryngologists and departments of ear, nose, and throat in all regions of Denmark. At referral, all patients had a CT scan of the nose and sinuses to evaluate sinonasal anatomy and pathology. All patients were screened with the MiniMental State Examination [22] and Major Depression Inventory (MDI) [23]. After clinical examination, medical history, and olfactory testing with Sniffin' Sticks extended olfactory test (Burghart Messtechnik GmbH, Wedel, Germany) [24], a clinical diagnosis with suspected cause of olfactory loss was established [25]. The diagnoses fell in 6 major categories: sinonasal, post-infectious, post-traumatic, congenital, iatrogenic, or idiopathic. Patients with suspected idiopathic, congenital, or post-traumatic cause of anosmia received a 3 Tesla (T) MRI scan of the brain with a customized olfactory sequence at the Department of Radiology, Aarhus University Hospital. Among the patients with sinonasal, post-infectious and iatrogenic olfactory loss, only patients with additional neurological symptoms such as headache, vertigo, slightly increased depression screening score, or history of cognitive changes after olfactory loss, received the $3 \mathrm{~T}$ MRI scan, in order to rule out central causes of olfactory loss.

From June 2018 through November 2019, we included 107 consecutive patients fulfilling the criteria for a $3 \mathrm{~T}$ MRI scan. Patients with idiopathic olfactory loss were compared with patients suffering from other common causes of acquired olfactory loss.

MRI and Assessment of Abnormalities

All patients were scanned with a Siemens MAGNETOM Skyra 3 Tesla MRI scanner (Siemens, Erlangen, Germany) using a 

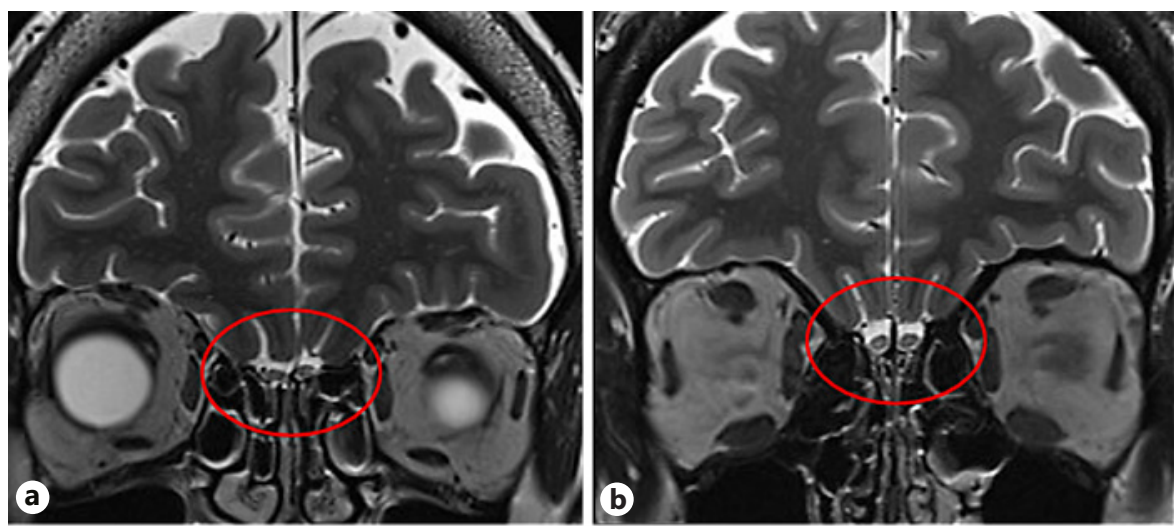

Fig. 1. Variants of normal shapes of the olfactory bulbs: a Oval. b Round. Note the central hyperintensity in both olfactory bulbs; this is called an olfactory ventricle, which is a remnant of an embryologic cavity in the olfactory bulb [32]. c Triangular. d Bean; this shape also covers boat shape or banana shape.
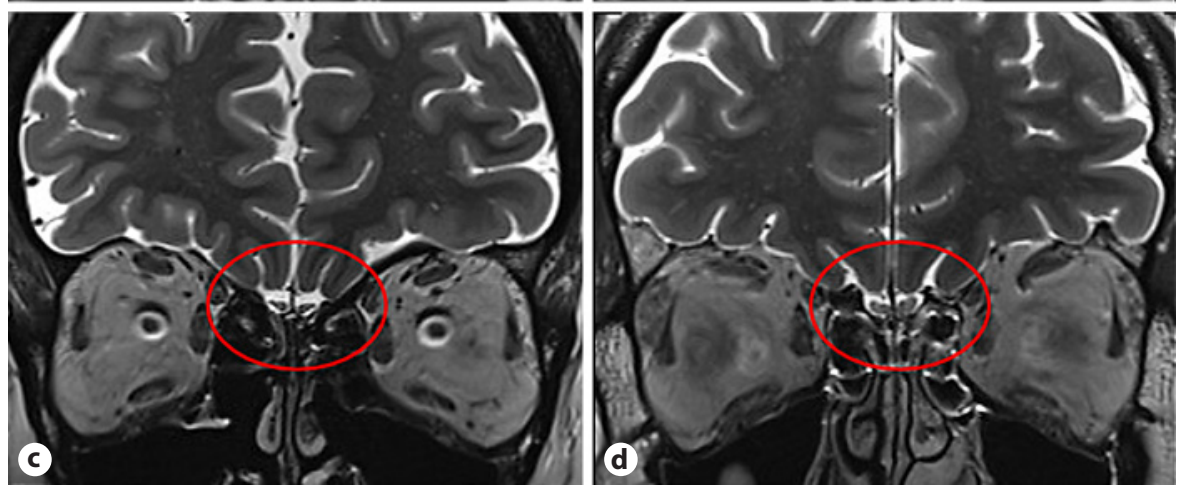

32-channel head coil. The MRI protocol comprised the following structural sequences: sagittal T1 3D MP2RAGE (TR $=5,000 \mathrm{~ms}$, $\mathrm{TE}=2.98 \mathrm{~ms}$, FOV $=256 \mathrm{~mm}$, slice thickness $=1.0 \mathrm{~mm})$, sagittal T2 3D space FLAIR $(\mathrm{TR}=5,000 \mathrm{~ms}, \mathrm{TE}=388 \mathrm{~ms}, \mathrm{FOV}=256 \mathrm{~mm}$, slice thickness $=1.0 \mathrm{~mm})$, coronal T2 TSE $(\mathrm{TR}=9,000 \mathrm{~ms}$, TE $=$ $113 \mathrm{~ms}$, flip angle $=145^{\circ}, \mathrm{FOV}=120 \mathrm{~mm}$, slice thickness $=1.4$ $\mathrm{mm})$, and axial T2 TSE $(\mathrm{TR}=5,260 \mathrm{~ms}, \mathrm{TE}=82 \mathrm{~ms}$, flip angle $=$ $150^{\circ}, \mathrm{FOV}=120 \mathrm{~mm}$, slice thickness $=2.0 \mathrm{~mm}$ ). In addition, we applied an axial DWI sequence $(\mathrm{TR}=5,700 \mathrm{~ms}$, TE1 $=64 \mathrm{~ms}$, $\mathrm{TE} 2=100 \mathrm{~ms}$, flip angle $=180^{\circ}, \mathrm{FOV}=220 \mathrm{~mm}$, slice thickness $=$ $4.0 \mathrm{~mm}$ ).

All MRI scans were evaluated by a trained neuroradiologist (R.B.D., author) for WMHs (Fazekas score) [26], infarctions, GCA score [27], MTA score [28], intracranial tumours, and other intracranial abnormalities. WMHs and lacunar infarctions were defined according to neuroimaging standards for small vessel disease [29]. Also, the shape of the OBs was assessed on the T2-weighted coronal sequence and sagittal reconstructed images parallel to the olfactory tract. The OB shapes were divided into the following categories: oval, round, triangular, boat, bean, banana, flat, scattered/ irregular and missing, or severely hypoplastic (examples are shown in Fig. 1-3). In addition, we categorized anatomical normal variations, including duplications and split bulbs. Categorization of the OBs according to shape is difficult and subjective when it comes to normal anatomical variations. We have interpreted oval, round, triangular, and bean/boat/banana shapes as normal variants of $\mathrm{OB}$ shape [30], which may reflect the different maturation stages in infancy [31]. Atrophy was interpreted as flattened OB, according to previous literature [32].

The bulb shapes were compiled into 5 categories:

MRI in Idiopathic Olfactory Loss
1. Normal (variants of normal shapes: Oval, round, triangular, boat, bean, and banana).

2. Atrophy (flat).

3. Scattered (irregular).

4. None (or severely hypoplasia).

5. Various; for example, split, duplication (normal anatomical variations outside category 1 ).

\section{Statistics}

Statistical analyses were completed using JMP (SAS Institute, Cary, NC, USA) software, version 14.0. Pearson's $\chi^{2}$ test was used for evaluating differences in categorical variables between all groups, while Fisher's exact test was used for $2 \times 2$ contingency tables. Normal distribution was assessed using QQ-plots and Shapiro-Wilk W test. In parametric data, mean values were compared using the Student's $t$ test and presented as means with 95\% confidence intervals $(95 \% \mathrm{CI})$. The $\alpha$ level of statistical significance was set at 0.05 . The $\mathrm{OB}$ shapes were assessed by a single rater (R.B.D.) and repeated for all subjects, blinded to previous ratings, and the intra-rater reliability (repeatability) was tested using the Cohen's kappa coefficient.

\section{Results}

Of the 107 consecutive patients fulfilling our criteria for a 3 T MRI, 88 patients had acquired olfactory loss and were included for comparative analyses, see Table 1. Of 

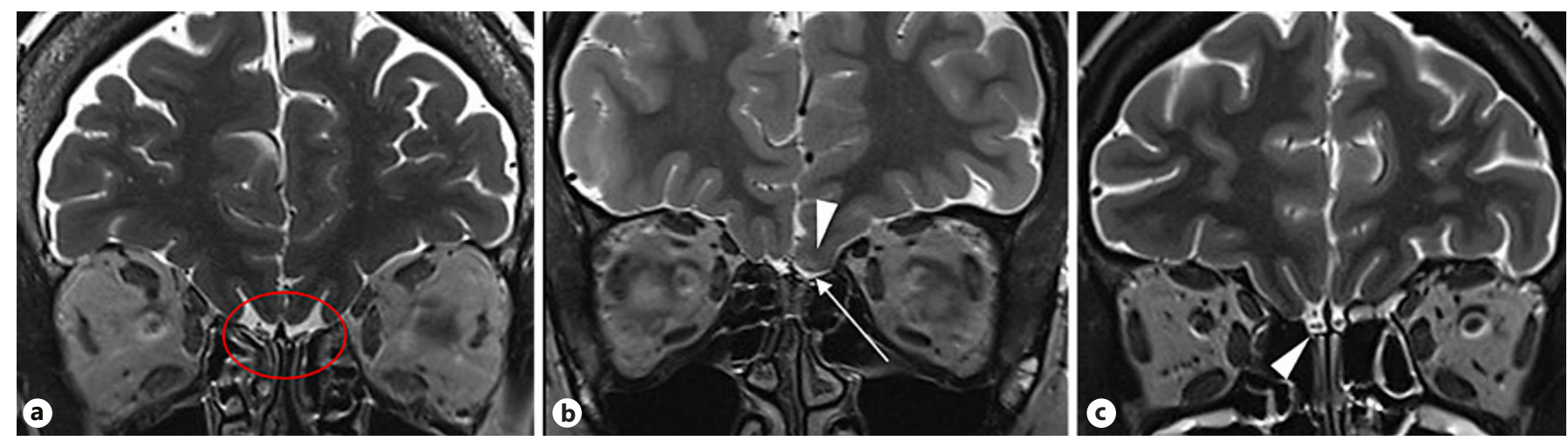

Fig. 2. Other shapes of olfactory bulbs. a Flat, here interpreted as consistent with atrophy. b None (missing); consistent with congenital anosmia. The olfactory groove is flat and contains no olfactory bulb or olfactory tract (arrow). Note also the hypoplasia of the olfactory sulcus (arrowhead). c Split or duplicated olfactory bulb (arrowhead). A blood vessel is seen just above the olfactory bulb.

Fig. 3. Scattered and/or irregular olfactory bulbs as a consequence of previous traumatic head injury. a Coronal T2-weighted image shows scattered olfactory bulb (arrow) and a small substance loss of the adjacent parenchyma with surrounding gliosis. The bilateral substance loss is also shown on the axial FLAIR image below (arrowheads). b Coronal T2-weighted image shows scattered olfactory bulb (arrow) and severe substance loss of the adjacent parenchyma with surrounding gliosis (arrowheads). The severe substance loss is also shown on the axial FLAIR image below (arrowhead).
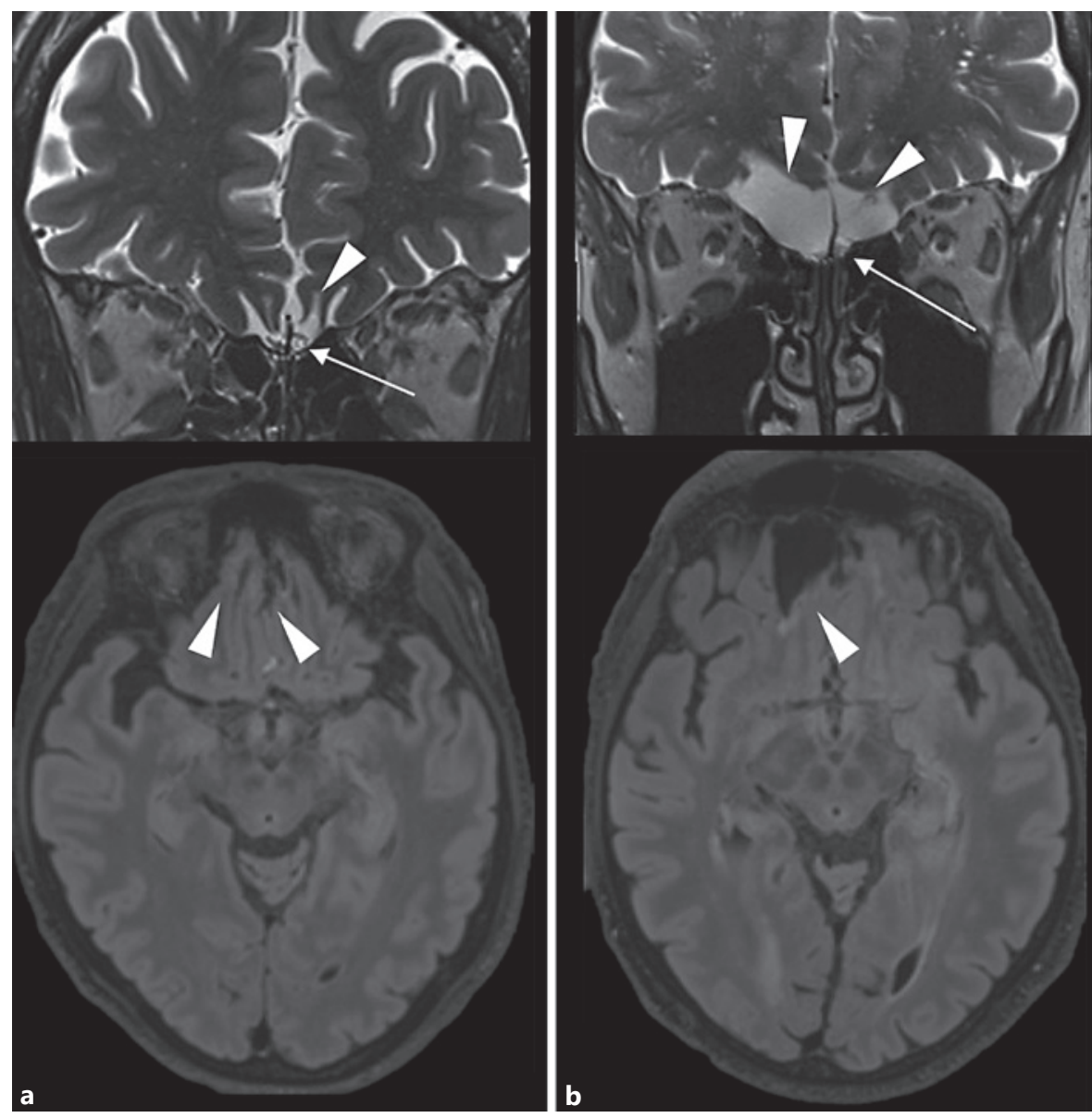

these, 51 were classified idiopathic, 7 sinonasal, 14 postinfectious, 13 post-traumatic, and 3 iatrogenic. The remaining 19 patients had both clinical and MRI findings (i.e., missing or severely hypoplastic OBs), consistent with congenital olfactory deficits, and were excluded from further analyses, as both the clinical presentation and age was not comparable to patients with acquired olfactory loss. Due to limited statistical power from small groups, the si- 
Table 1. Demographics and clinical scores

\begin{tabular}{lllll}
\hline & All causes $(n=88)$ & $\begin{array}{l}\text { Idiopathic olfactory } \\
\text { loss }(n=51)\end{array}$ & $\begin{array}{l}\text { Other causes of olfactory } \\
\text { loss }(n=37)\end{array}$ & $\begin{array}{l}\text { Difference (idiopathic } \\
\text { vs. other) }\end{array}$ \\
\hline Age (mean, 95\% CI) & $57.0(54.2 ; 59.9)$ & $57.6(53.7 ; 61.4)$ & $56.3(51.8 ; 60.7)$ & 0.6541 \\
Gender (\% female) & 59.1 & 58.8 & 59.5 & 0.9522 \\
Smoking, \% (current/previous/never) & $14 / 35 / 51$ & $8 / 43 / 49$ & $22 / 24 / 54$ & 0.0705 \\
Allergy, \% & 25.3 & 30.0 & 18.9 & 0.2837 \\
Cause of olfactory loss, $n$ & - & - & Sinonasal: 7 & - \\
& & & Post-viral: 14 & Post-traumatic: 13 \\
& & & $14.1(12.5 ; 15.8)$ & 0.9908 \\
latrogenic: & $1.7(1.2 ; 2.1)$ & 0.9010 \\
ThI olfactory score & $14.1(13.0 ; 15.2)$ & $14.1(12.6 ; 15,6)$ & $6.0(5.3 ; 6.7)$ & 0.5472 \\
Discrimination score & $1.6(1.3 ; 1.9)$ & $1.6(1.3 ; 2.0)$ & $6.4(5.3 ; 7.6)$ & 0.6645 \\
Identification score & $6.2(5.7 ; 6.7)$ & $6.3(5.6 ; 7.1)$ & $13.1(8.8 ; 17.5)$ & $0.0014^{*}$ \\
MDI score & $6.3(5.6 ; 6.9)$ & $6.1(5.3 ; 6.9)$ & $28.2(27.8 ; 28.6)$ & $0.0285^{*}$ \\
MMSE score & $8.6(6.4 ; 10.8)$ & $5.3(3.7 ; 6.9)$ & $27.1(20.9 ; 33.2)$ & $0.0019^{*}$ \\
SNOT-22 score & $28.5(28.3 ; 28.8)$ & $28.8(28.5 ; 29.1)$ & $16.0(13.0 ; 19.0)$ & \\
\hline
\end{tabular}

95\% Cl, 95\% confidence interval; MDI, Major Depression Inventory score; MMSE, Mini-Mental State Examination; SNOT-22, SinoNasal Outcome Test; TDI, Olfactory threshold, Discrimination, and Identification score (mean, 95\% CI). ${ }^{*}$ Indicates significant differences between groups.

Table 2. MRI abnormalities and scores

\begin{tabular}{|c|c|c|c|c|}
\hline & $\begin{array}{l}\text { All causes } \\
(n=88)\end{array}$ & $\begin{array}{l}\text { Idiopathic olfactory } \\
\text { loss }(n=51)\end{array}$ & $\begin{array}{l}\text { Other causes of } \\
\text { olfactory loss }(n=37)\end{array}$ & $\begin{array}{l}\text { Difference } \\
\text { (idiopathic vs. other) }\end{array}$ \\
\hline \multicolumn{5}{|l|}{ Fazekas score } \\
\hline $0-1, n(\%)$ & $71(81)$ & $39(76)$ & $32(86)$ & \\
\hline $2-3, n(\%)$ & $17(19)$ & $12(24)$ & $5(14)$ & \\
\hline Mean & $0.86(0.68 ; 1.05)$ & $0.94(0.67-1.21)$ & $0.76(0.61 ; 1.07)$ & 0.2977 \\
\hline \multicolumn{5}{|l|}{ GCA score } \\
\hline $0-1, n(\%)$ & $28(32)$ & $16(31)$ & $12(32)$ & \\
\hline $2-3, n(\%)$ & $60(68)$ & $35(69)$ & $25(68)$ & \\
\hline Mean & $0.88(0.72 ; 1.03)$ & $0.90(0.70 ; 1.11)$ & $0.84(0.61 ; 1.07)$ & 0.6748 \\
\hline \multicolumn{5}{|l|}{ MTA score } \\
\hline$<1.5, n(\%)$ & 74 (84) & $42(82)$ & $32(86)$ & \\
\hline$\geq 1.5, n(\%)$ & $14(16)$ & $9(18)$ & $5(14)$ & \\
\hline Mean & $0.65(0.48 ; 0.81)$ & $0.67(0.44 ; 0.90)$ & $0.62(0.38 ; 0.86)$ & 0.7851 \\
\hline Lacunar infarction, $n$ (\%) & $5(5.7)$ & $3(5.9)$ & $2(5.4)$ & 0.9247 \\
\hline \multicolumn{5}{|l|}{ Bulb configuration, $n(\%)$} \\
\hline Normal & $58(65.9)$ & $34(66.7)$ & $24(64.9)$ & $0.0269^{*}$ \\
\hline Atrophy & $20(22.7)$ & $14(27.5)$ & $6(16.2)$ & \\
\hline Scattered & $6(6.8)$ & $0(0 \%)$ & $6(16.2)$ & \\
\hline None & $2(2.3)$ & $1(2.0)$ & $1(2.7)$ & \\
\hline Various & $2(2.3)$ & $2(3.9)$ & $0(0)$ & \\
\hline
\end{tabular}

GCA, global cortical atrophy; MTA, medial temporal lobe atrophy; MRI, magnetic resonance imaging.

nonasal, post-infectious, post-traumatic, and iatrogenic causes were pooled in 1 group, termed "other causes."

The demographic variables and olfactory test scores were comparable between the idiopathic group and the group with other acquired causes. The SNOT-22, MDI, and MMSE scores were higher in the "other causes" group compared with the idiopathic group. As the other causes include sinonasal olfactory loss, the increased SNOT22 score in this group was expected. The higher MDI scores in "other causes" was a contributing cause for referral to 
MRI due to known co-occurrence of olfactory loss and mild depression in olfactory groove meningiomas [33]. None of the patients in either group had MDI scores indicating major depression. All MMSE scores were within the normal range. For all patients, cerebral changes were evaluated with Fazekas score (white matter lesions), GCA score, and MTA score, see Table 2.

Fazekas scores were dichotomized into $0-1$ and $2-3$, considering the beginning confluency of WMHs as a marker of small vessel disease [25]. GCA scores were dichotomized into $0-1$ and $2-3$, considering a distinction between no/mild atrophy and moderate to severe atrophy, the most appropriate for our study population with a mean age of 57 years [26]. Left/right average MTA scores $\geq 1.5$ were regarded as representing pathological MTA $<75$ years $[28,34]$. Statistical analyses showed no significant differences in the selected structural imaging scores between patients with idiopathic olfactory loss and patients with other causes of olfactory loss (see Table 2).

The distribution of $\mathrm{OB}$ configurations differed between the groups, mostly driven by the scattered/irregular OBs, which was primarily found in post-traumatic patients (5/13 patients) and a single occurrence in a patient with post-infectious olfactory loss. As such, the occurrence of scattered/irregular OBs has a specificity of $98.6 \%$ $(74 /[74+1] \times 100)$ and a sensitivity of $38.5 \%(74 /[74+8]$ $\times 100)$ for post-traumatic aetiology of olfactory loss.

The intra-rater reliability (repeatability) of OB shape assessment was strong (Cohen's kappa $=0.87 ; 95 \% \mathrm{CI}=$ $0.79-0.95 ; n=107)$. The repeatability remained strong after exclusion of 19 clinical cases with congenital anosmia (Cohen's kappa $=0.81 ; 95 \% \mathrm{CI}=0.69-0.93 ; n=88$ ).

Benign incidental findings MRI findings included an arachnoid cyst $(n=1)$, a choroid plexus xanthogranuloma $(n=1)$, a pituitary cyst $(n=1)$, unspecific FLAIR hyperintensity $(n=1)$, and a venous abnormality combined with myelination defect $(n=1)$. Furthermore, grey matter trauma sequelae (all with scattered/irregular OBs) were identified in 4 of 13 patients with post-traumatic olfactory loss. Some of these trauma sequelae were subtle and required both good image quality and attention to the affected cortical regions by the neuroradiologist. No brain tumours were identified in this study population.

\section{Discussion}

Key Findings

We found no significant differences in WMHs (Fazekas score), GCA score, or MTA score in patients with olfactory loss classified as idiopathic compared to other aetiologies. The shape of the OB differed between patients with idiopathic olfactory loss and patients with other causes of olfactory loss. The significant difference in OB shapes was driven by a high occurrence of shattered/irregular bulbs in patients with post-traumatic olfactory loss.

The occurrence of scattered/irregular OBs was specific $(98.6 \%)$, but not sensitive $(38.5 \%)$, for post-traumatic olfactory loss aetiology along with grey/white matter trauma sequelae. Head trauma with subsequent olfactory loss often implies specific sequelae in the OBs, typically shearing/tearing of the olfactory nerve fibres, and traumatic injury to the adjacent olfactory cortices [35]. MRI assessment of the $\mathrm{OB}$ integrity and possible traumatic injury of the brain parenchyma, for example, substance loss with surrounding gliosis, typically found in the frontobasal cortex and the temporal poles, may support the diagnosis of olfactory loss due to previous head trauma. 3T MRI may be superior to $1.5 \mathrm{~T}$ MRI in this regard.

\section{Use of MRI to Identify Tumours in the Diagnostic} WorkUp of Olfactory Loss

No tumours were identified in the current study. In a large study on 750 patients with smell loss with mixed aetiology (post-infectious [26\%], idiopathic [22\%], head trauma [18\%], and sinonasal disease [15\%]), the incidence of brain tumours was only $0.3 \%$. However, systematic use of neuroimaging was not described for this population, while the incidence may be underestimated [34].

Olfactory groove meningiomas are the most commonly described intracranial tumour causing smell loss and are slow-growing with discrete, non-specific symptoms such as slowly progressing smell disturbances, headaches, visual disturbances, seizures, or neuropsychiatric symptoms (e.g., depression, personality changes, or declining cognitive function) $[36,37]$. While these symptoms are relevant to assess, slowly progressing smell disturbances may be the only symptom.

A US-based study on cost-effectiveness on performing MRI in patients with idiopathic olfactory disorders concluded that MRI should be performed in this patient group solely based on the risk of missing intracranial tumours [5]. Here, intracranial tumours were found in $6 / 122$ patients, including meningioma $(n=3)$, clival mass $(n=2)$, and pituitary adenoma $(n=1)$. Two of these meningiomas were not incidental findings and had already been previously diagnosed. However, the cost-effectiveness assessment was based on the US health care system, where the medical malpractice settlements for misdiagnosis leading to neurological damage had a median settle- 
ment of USD 6,000,000 [5]. As such, this cost-effectiveness calculation differs from most other countries and has little external validity. However, tumours often constitute a concern in patients with olfactory loss and, although rare, should be a differential diagnosis in the clinical workup of relevant patients.

\section{Other Potentially Relevant MRI Information in \\ Relation to Olfactory Loss}

Although we found no significant differences in typical MRI measures of small vessel disease or degenerative diseases with olfactory loss classified as idiopathic compared to other aetiologies, we cannot preclude that other structural findings may add to a clinical diagnosis with known olfactory symptoms. A recent study indicates that $\mathrm{OB}$ surroundings can potentially help to distinguish Parkinson's disease from non-Parkinsonian olfactory dysfunction [38].

\section{$1.5 \mathrm{~T}$ or $3 \mathrm{~T} \mathrm{MRI}$ ?}

It has previously been established that MRI can be relevant in the clinical workup of olfactory loss for other reasons than identifying intracranial tumours. While 1.5T MRI is sufficient for the diagnosis of intracranial tumours causing the olfactory loss, 3T MRI may more sufficiently be used to assess the OB shape and volume due to higher signal-to-noise ratio and increased spatial resolution. Besides being a possible marker of olfactory function, the $\mathrm{OB}$ volume can aid in establishing the prognosis and possible effects of olfactory training [39]. The evaluation of olfactory structures is also important in the diagnosis of congenital anosmia in, for example, Kallmann syndrome [40,41], as timely diagnosis and treatment will induce puberty, leading to improved sexual, bone, metabolic, and psychological health [42]. Another study has successfully shown that a brain-morphology-based algorithm with 1.5T MRI can predict the diagnosis of anosmia, hyposmia, or normosmia with a $62.3 \%$ accuracy following head trauma in patients, indicating that $1.5 \mathrm{~T}$ is useful in the clinical assessment of olfactory disorders [43]. Hence, the choice between a 1.5T and 3T MRI depends on the indication for MRI acquisition. Considering the high number of patients with long lasting olfactory loss following the COVID-19 pandemic [44], the economic strain on health care systems could benefit from thorough consideration of MRI indications, as 1.5T MRI is often cheaper and more accessible than 3T MRI.

\section{Limitations}

Assessment of $\mathrm{OB}$ configuration was based on visual inspection; the inspection was performed by a single neu- roradiologist to avoid inter-rater disagreement with excellent intra-rater reliability. We did not perform volumetric OB measurement. Manual measurement of $\mathrm{OB}$ volume can be a relevant tool to quantify $\mathrm{OB}$ volume for comparison with normative values and for follow-up [28], but a time-consuming procedure in daily clinical practice. To our knowledge, automated segmentation of $\mathrm{OB}$ is not yet available for daily clinical use.

The criteria for ordering MRI in the diagnostic workup of non-idiopathic aetiologies in the current study included symptoms of headache and/or elevated MDI scores. As headache is among the diagnostic criteria for chronic rhinosinusitis, patients in the sinonasal group were referred to MRI if endoscopic findings did not reveal a plausible cause of headache. The slightly elevated MDI scores in the "other causes" group did not include any scores indicating major depression. However, these factors of clinical relevance for conducting the scans may affect the external validity of our MRI findings.

The current study is based on patients with mixed causes of olfactory loss, where some of the non-idiopathic groups are relatively small in sizes. Although a larger study population would increase statistical power, the current dataset enabled a classification of different bulb morphologies which have undergone test-retest reliability assessment. Larger studies on bulb morphologies in different olfactory loss etiologies are warranted to address further sub-classifications of bulb morphology.

\section{Conclusions}

Assessment of WMHs, GCA, and MTA atrophy on MRI in patients with olfactory loss did not aid in differentiating patients with idiopathic olfactory loss from other causes of olfactory loss and is not warranted in the diagnostic process of acquired olfactory loss alone. Assessment of the OB configuration with MRI may aid in the diagnostic evaluation of congenital and post-traumatic olfactory dysfunction.

We suggest that MRI of the brain with $\mathrm{OB}$ sequences should be considered in the diagnostic evaluation of olfactory loss in cases of clinically suspected congenital anosmia, post-traumatic olfactory dysfunction, and in idiopathic olfactory loss where a central component (e.g., tumour) should be ruled out. The choice of 1.5 or 3T MRI depends on diagnostic demands and availability. 


\section{Acknowledgements}

We would like to thank professor Thomas Hummel, Technische Universität Dresden, Germany, for his valuable input and feedback on the study and Dovile Stankevice for her large contribution with data collection.

\section{Statement of Ethics}

All patients consented to register clinical data in a REDCap database, and the study was approved by the regional data protection authority. As the study is retrospective analysis, ethics approval was not warranted. All patients have given their written informed consent.

\section{Conflict of Interest Statement}

The authors have no conflicts of interest.

\section{Funding Sources}

None of the authors received funding for the study. The first author was partly funded by the Health Research Fund of Central Denmark Region and VELUX FONDEN. The sponsors had no say, roles, or responsibilities in relation to the study, including (but not limited to) the study design, data collection, management, analysis, interpretation of data, writing of the manuscript, or the decision to publish.

\section{Author Contributions}

The idea for the project was conceived by A.F., T.O., and R.B.D. R.B.D. conducted neuroradiologic evaluation of all MRI scans; A.F. analysed the data; A.F. wrote the initial draft for the manuscript; A.F., T.O., and R.B.D. contributed to a subsequent review of the manuscript.

\section{Data Availability Statement}

Due to GDPR restrictions, patient data cannot be made publicly available. Further information on the data and anonymized elements of the data can be acquired from the corresponding author.

\section{References}

1 Damm M, Temmel A, Welge-Lüssen A, Eckel HE, Kreft MP, Klussmann JP, et al. [Olfactory dysfunctions. Epidemiology and therapy in Germany, Austria and Switzerland]. HNO. 2004;52:112-20.

2 Rombaux P, Duprez T, Hummel T. Olfactory bulb volume in the clinical assessment of olfactory dysfunction. Rhinology. 2009;47:3-9.

3 Hummel T, Whitcroft KL, Andrews P, Altundag A, Cinghi C, Costanzo RM, et al. Position paper on olfactory dysfunction. Rhinology. 2016;56:1-30.

4 Higgins TS, Lane AP. What is the best imaging modality to investigate olfactory dysfunction in the setting of normal endoscopy? Laryngoscope. 2014;124:4-5.

5 Decker JR, Meen EK, Kern RC, Chandra RK. Cost effectiveness of magnetic resonance imaging in the workup of the dysosmia patient. Int Forum Allergy Rhinol. 2012;3:56-61.

6 Hawkes C. Olfaction in neurodegenerative disorder. Mov Disord. 2003;18:364-72.

7 Witoonpanich P, Cash D, Shakespeare TJ, Yong K, Nicholas JM, Omar R, et al. Olfactory impairment in posterior cortical atrophy. J Neurol Neurosurg Psychiatry. 2013;84:58890.

8 Haehner A, Masala C, Walter S, Reichmann $\mathrm{H}$, Hummel T. Incidence of Parkinson's disease in a large patient cohort with idiopathic smell and taste loss. J Neurol. 2019;266:33945.

9 Wattendorf E, Welge-Lüssen A, Fiedler K, Bilecen D, Wolfensberger M, Fuhr P, et al. Olfactory impairment predicts brain atrophy in
Parkinson's disease. J Neurosci. 2009;29: 15410-3.

10 Lee EY, Eslinger PJ, Du G, Kong L, Lewis MM, Huang X. Olfactory-related cortical atrophy is associated with olfactory dysfunction in Parkinson's disease. Mov Disord. 2014;29:1205-8.

11 Bitter T, Brüderle J, Gudziol H, Burmeister HP, Gaser C, Guntinas-Lichius O. Gray and white matter reduction in hyposmic subjects: a voxel-based morphometry study. Brain Res. 2010;1347:42-7.

12 Bitter T, Gudziol H, Burmeister HP, Mentzel HJ, Guntinas-Lichius O, Gaser C. Anosmia leads to a loss of gray matter in cortical brain areas. Chem Senses. 2010;35:407-15.

13 Bitter T, Gudziol H, Burmeister HP, Mentzel HJ, Guntinas-Lichius O, Gaser C. Anosmia leads to a loss of gray matter in cortical brain areas. Chem Senses. 2010;35(5):407-15.

14 Yoshii F, Onaka H, Kohara S, Ryo M, Takahashi W. Association of smell identification deficit with Alzheimer's disease assessment scale-cognitive subscale, Japanese version scores and brain atrophy in patients with dementia. Eur Neurol. 2019;81:145-51.

15 Haehner A, Rodewald A, Gerber JC, Hummel T. Correlation of olfactory function with changes in the volume of the human olfactory bulb. Arch Otolaryngol Head Neck Surg. 2008;134(6):621-4

16 Smitka M, Abolmaali N, Witt M, Gerber JC, Neuhuber W, Buschhueter D, et al. Olfactory bulb ventricles as a frequent finding in magnetic resonance imaging studies of the olfactory system. NSC. 2009;162:482-5.
17 Lu R, Aziz NA, Reuter M, Stöcker T, Breteler MMB. Evaluation of the neuroanatomical basis of olfactory dysfunction in the general population. JAMA Otolaryngology Head Neck Surg. 2021;147:855-63.

18 Yildirim D, Altundag A, Tekcan Sanli DE, Bakir A, Eryurekli A, Alis D, et al. A new perspective on imaging of olfactory dysfunction: does size matter? Eur J Radiol. 2020;132: 109290.

19 Wardlaw JM, Hernández MCV, Muñoz Maniega S. What are white matter hyperintensities made of? J Am Heart Assoc. 2015;4: 001140.

20 Heinrich J, Vidal J-S, Simon A, Rigaud A-S, Hanon O, Epelbaum J, et al. Relationships between lower olfaction and brain white matter lesions in elderly subjects with mild cognitive impairment. J Alzheimers Dis. 2018;61:113341.

21 Zorzon M, Ukmar M, Bragadin LM, Zanier F, Antonello RM, Cazzato G, et al. Olfactory dysfunction and extent of white matter abnormalities in multiple sclerosis: a clinical and MR study. Mult Scler. 2000;6:386-90.

22 Folstein MF, Folstein SE, McHugh PR. "Minimental state". A practical method for grading the cognitive state of patients for the clinician. J Psychiatr Res. 1975;12:189-98.

23 Bech P, Rasmussen NA, Olsen LR, Noerholm $\mathrm{V}$, Abildgaard W. The sensitivity and specificity of the major depression inventory, using the present state examination as the index of diagnostic validity. J Affect Disord. 2001;66: $159-64$. 
24 Niklassen AS, Ovesen T, Fernandes H, Fjaeldstad AW. Danish validation of Sniffin' sticks olfactory test for threshold, discrimination, and identification. Laryngoscope. 2017;114: 1764-8.

25 Fjaeldstad A, Stankovic J, Onat M, Stankevice D, Ovesen T. Patients and experiences from the first Danish flavour clinic. Dan Med J. 2020;67(4):1-5.

26 Fazekas F, Chawluk JB, Alavi A, Hurtig HI, Zimmerman RA. MR signal abnormalities at 1.5 T in Alzheimer's dementia and normal aging. AJR Am J Roentgenol. 1987;149:351-6.

27 Pasquier F, Leys D, Weerts JGE, Mounier-Vehier F, Barkhof F, Scheltens P. Inter-and intraobserver reproducibility of cerebral atrophy assessment on mri scans with hemispheric infarcts. Eur Neurol. 1996;36:268-72.

28 Scheltens P, Leys D, Barkhof F, Huglo D, Weinstein HC, Vermersch P. Atrophy of medial temporal lobes on MRI in "probable区 $\mathrm{Al}$ zheimer's disease and normal ageing: diagnostic value and neuropsychological correlates. J Neurol Neurosurg Psychiatry. 1992;55: $967-72$.

29 Wardlaw JM, Smith EE, Biessels GJ, Cordonnier C, Fazekas F, Frayne R, et al. Neuroimaging standards for research into small vessel disease and its contribution to ageing and neurodegeneration. Lancet Neurol. 2013;12: $822-38$.

30 Rombaux P, Duprez T, Hummel T. Olfactory bulb volume in the clinical assessment of olfactory dysfunction. Rhinology. 2009;47:3-9.
31 Schneider JF, Floemer F. Maturation of the olfactory bulbs: MR imaging findings. AJNR Am J Neuroradiol. 2009;30:1149-52.

32 Chung MS, Choi WR, Jeong HY, Lee JH, Kim JH. MR imaging-based evaluations of olfactory bulb atrophy in patients with olfactory dysfunction. AJNR Am J Neuroradiol. 2018; 39:532-7.

33 Kessler RA, Loewenstern J, Kohli K, Shrivastava RK. Is psychiatric depression a presenting neurologic sign of meningioma? A critical review of the literature with causative etiology. World Neurosurg. 2018;112:64-72.

34 Claus JJ, Staekenborg SS, Holl DC, Roorda JJ, Schuur J, Koster P, et al. Practical use of visual medial temporal lobe atrophy cut-off scores in Alzheimer's disease: validation in a large memory clinic population. Eur Radiol. 2017 Aug;27(8):3147-55.

35 Howell J, Costanzo RM, Reiter ER. Head trauma and olfactory function. World J Otorhinolaryngol Head Neck Surg. 2018;4:39-45.

36 Bakay L. Olfactory meningiomas. The missed diagnosis. Jama. 1984;251(1):53-5.

37 Niklassen AS, Jørgensen RL, Fjaeldstad AW. Olfactory groove meningioma with a 10-year history of smell loss and olfactory recovery after surgery. BMJ Case Rep. 2021;14(8): e244145.

38 Tremblay C, Mei J, Frasnelli J. Olfactory bulb surroundings can help to distinguish Parkinson's disease from non-parkinsonian olfactory dysfunction. Neuroimage Clin. 2020;28: 102457.
39 Rombaux P, Huart C, Deggouj N, Duprez T, Hummel T. Prognostic value of olfactory bulb volume measurement for recovery in postinfectious and posttraumatic olfactory loss. Otolaryngol Head Neck Surg. 2012;147: 1136-41.

40 Huart C, Meusel T, Gerber J, Duprez T, Rombaux P, Hummel T. The depth of the olfactory sulcus is an indicator of congenital anosmia. AJNR Am J Neuroradiol. 2011;32:19114.

41 Levy LM, Degnan AJ, Sethi I, Henkin RI. Anatomic olfactory structural abnormalities in congenital smell loss: magnetic resonance imaging evaluation of olfactory bulb, groove, sulcal, and hippocampal morphology. J Comput Assist Tomogr. 2013;37:650-7.

42 Young J, Xu C, Papadakis GE, Acierno JS, Maione L, Hietamäki J, et al. Clinical management of congenital hypogonadotropic hypogonadism. Endocr Rev. 2019;40:669-710.

43 Lötsch J, Reither N, Bogdanov V, Hähner A, Ultsch A, Hill K, et al. A brain-lesion pattern based algorithm for the diagnosis of posttraumatic olfactory loss. Rhinology. 2015;53(4): 365-70.

44 Schwab J, Jensen CD, Fjaeldstad AW. Sustained chemosensory dysfunction during the COVID-19 Pandemic. Orl. 2021;83:1-10. 